\title{
Fagotti Score
}

National Cancer Institute

\section{Source}

National Cancer Institute. Fagotti Score. NCI Thesaurus. Code C162368.

A laparoscopy-based score to predict the chances of optimal cytoreductive surgery in advanced ovarian cancer patients. It consists of seven criteria (omental cake, peritoneal carcinosis, diaphragmatic carcinosis, mesenteric retraction, bowel infiltration, stomach infiltration, and liver metastases), each assigned a predictive index value of 0-2. The total Fagotti score ranges from 0-14. 